\title{
Frequency and Associated Factors of Autonomic Dysfunction in Patients with Parkinson's Disease in Khartoum State
}

\author{
Abdel Mumin Sidahmed*, Husam A. M. Ali \\ Omdurman Teaching Hospital, Omdurman, Sudan \\ Email: *mumin.sidahmed@outlook
}

How to cite this paper: Sidahmed, A.M. and Ali, H.A.M. (2019) Frequency and Associated Factors of Autonomic Dysfunction in Patients with Parkinson's Disease in Khartoum State. Advances in Parkinson's Disease, 8, 63-74.

https://doi.org/10.4236/apd.2019.84006

Received: August 18, 2019

Accepted: October 29, 2019

Published: November 1, 2019

Copyright $\odot 2019$ by author(s) and Scientific Research Publishing Inc. This work is licensed under the Creative Commons Attribution International License (CC BY 4.0).

http://creativecommons.org/licenses/by/4.0/

\begin{abstract}
Background: Autonomic dysfunction in idiopathic Parkinson disease is a frequent and disabling complication, with an estimated prevalence of $47 \%$ and has a significant impact on the patient's quality of life. Objectives: The main objective of this study was to determine the frequency of autonomic dysfunction among Sudanese Parkinson patients and identify possible risk factors attribute to develop autonomic dysfunction and to assess the extent to which the progression of dysautonomia affects activities of daily living, health-related quality of life. Methods: In this descriptive perspective, cross-sectional hospital-based study, 51 patients were studied using standardized questionnaire including history and clinical examination. Results: A total of 51 patients have been examined: male to female ratio 1.5:1; mean age $55 \pm 5$ years; Parkinson disease duration, $7 \pm 2$ years. $47 \%$ of the patients had one or more symptoms of autonomic dysfunction with mean age $59 \pm 10$. Constipation and bloating were the most common symptoms where sweating abnormality was the least symptoms to observe. The symptom of autonomic dysfunction has been worse with disease progression in $50 \%$ of the patients and $47 \%$ of the patients reported that both motors and autonomic dysfunction symptoms were causing disability than autonomic dysfunction symptoms alone. Conclusions: The study demonstrates that autonomic dysfunction is not only common in Parkinson Disease, but it increases in severity with increasing disease stages. Older age with long disease duration was also considered along with advanced disease stages strong factors determining the presence of autonomic dysfunction. The study recommends that symptoms of autonomic dysfunction survey be a routine aspect of the evaluation of Parkinson disease patients, especially with advanced age.
\end{abstract}

\section{Keywords}

Idiopathic Parkinson Disease, Autonomic Dysfunctions, Associated Factors, 
Frequency

\section{Introduction}

Parkinson's disease (PD) is a progressive neurogenerative illness. It is first described by James Parkinson in his classic 1817 monograph, "An Essay on the Shaking Palsy" [1]. Patients with Parkinson's disease (PD) have a combination of motor impairment, cognitive dysfunction and autonomic failure during their illness. It affects $70 \%$ to $80 \%$ of patients [2] and causes significant morbidity and discomfort for the patients and caregiver. The frequency of Parkinson's disease (PD) is 1.5 times more in men than women probably due to the effect of estrogen on dopaminergic neurons and pathways in the brain [3]. Association between cigarette smoking and PD has been observed consistently during the past 30 years [4] in Cohort studies, in which data on smoking were obtained before the onset of $\mathrm{PD}$, and provide evidence for the possible protective effect of smoking. Relationship between obesity and the occurrence of PD is unknown. But a recent meta-analysis [5] of 12 case-control studies showed that PD patients had a significantly lower BMI than controls. Autonomic dysfunction symptoms in PD include derangements of cardiovascular regulation, particularly, orthostatic hypotension and gastrointestinal disorders, and bladder abnormalities [6]. The involvement of the cardiovascular autonomic system in PD is very important. The most frequent symptom is orthostatic hypotension, occurring in over half of the patients [7] regarding involvement of gastrointestinal system. Constipation is one of the most common symptoms among Parkinson Disease [8]. Sometimes it is even considered to be a potential premotor marker of PD and it has been correlated with the presence of incidental Lewy bodies in the bowel in a population without PD [9]. Bladder dysfunction is another common symptom of PD. Studies report that storage symptoms are present in $57 \%-83 \%$ of patients, whereas voiding symptoms are seen in $17 \%-27 \%$ patients and nocturnal urination is the most common complaint in $>60 \%$ patients with $\mathrm{PD}$ [10]. The care of a Parkinson disease patient with autonomic dysfunction depends on its early recognition and pointed therapy including coordinated care between the neurologist and appropriate subspecialist. Some drugs may be useful to treat a few of these symptoms manifestation. Although there is autonomic dysfunction in most patients with Parkinson's disease (PD), they are often unrecognized because many patients remain relatively asymptomatic in the early stage. This research was focused on the frequency, associative factors, and negative impacts of autonomic dysfunction in patient's life.

\section{Materials and Methods}

\subsection{Study Design and Area}

The study was a prospective, cross-sectional hospital-based study. The partici- 
pants in this study were enrolled from neurology refer Clinic in Khartoum state at the following tertiary hospitals: Omdurman teaching hospital, Bashar teaching hospital, soba university hospital and national neurology center. All patients were diagnosed with idiopathic Parkinson Disease (PD) by neurology specialists and in a regular follow up.

\subsection{Study Population and Sampling}

All Patients clinically diagnosed PD aged between 21 and 80 years old were eligible. Exclusion criteria included secondary Parkinsonism and atypical Parkinson disease presentation, movement disorders other than $\mathrm{PD}$, other causes of autonomic dysfunction which including autoimmune neuropathies, severe and complicated diabetes (presence of diabetic neuropathies, diabetic nephropathy and diabetic retinopathy), other conditions that might interfere with the reliable completion of clinical assessments. The size of the sample in this study was determined by total converge of the patients attending the neurological clinics in the study area.

\subsection{Assessment and Data Collection}

All information was collected by a face-to-face interview during a one-time interview in outpatient clinics using questionnaires. PD-non-specific data includes demographic information, lifestyle factors. Demographic information including age, sex, and marital status was collected during a clinical interview. Smoking and alcohol status, physical activity and disease information were assessed. PD-specific variables including age at onset (AAO), disease duration, family history of PD and current anti-parkinsonism medications were documented. There is many staging system to assess the disease activity in this study Disease stages were assessed with the Hoehn Yahr staging system (H-Y stage) [11].

Hoehn Yahr staging system rating is based upon examination of the patient which is the most common known evaluation of people with PD and was first described in 1967 (Hoehn and Yahr, 1967). It is a simple staging from 0 to 5 depending in the motor manifestations of $\mathrm{PD}$, to reflect the progression, and combines features of motor impairment and disability. It is well known and the tests are easily performed.

Screening of autonomic symptoms has been relied on detailed history taking and clinical examination and bedside test including for example vital signs, biometric measurement several standing and flat blood pressure, serial ECG and bedside ultrasound to assess bladder.

\subsection{Data Analysis}

Statistical analyses were performed with SPSS Statistics (version 25.0, SPSS Inc., Chicago, IL, USA). Continuous variables were given as means and standard deviation provided. Categorical variables were summarized by percentages. chi-square tests which have been used for categorical variables, $t$ tests for nor- 
mally distributed variables and Mann-Whitney tests for non-parametric variables for between-group comparisons. Logistic regression analysis was used to identify possible risk factors for autonomic dysfunction. $95 \%$ confidence intervals (CI) have been adopted. A P $<0.05$ was considered significant.

\subsection{Ethical Considerations}

The proposal of the study has been presented to the ethical Clearance committee of the Sudan Medical Specialization Board, and the Council of internal medicine and approved. Hospital directors and participants also have been informed about the purposes and objectives of the study and both verbally and written consents have been obtained.

\section{Result}

\subsection{Demographic and Clinical Data}

A total of 51 patients with PD were included in this study during the period from August 2017 to January 2018. 31 (60\%) patients were male and 20 (40\%) patients were female. The age of patients on presentation ranged from 40 to 80 years with mean age of $55.3 \pm 5$ the age groups were $41-50$ years counted 10 (20\%) patients, 51 - 60 years was $13(25 \%)$ and $61-70$ years was $16(31 \%)$ and above 70 years was 12 (24\%).

The onset of PD duration has been categorized into five categories; less than one year 11 (21\%) patients, two to five years 23 (45\%) patients, six to ten years $11(22 \%)$ patients and more than 11 years six $(12 \%)$ patients.

Severity of the disease has been assessed using $\mathrm{H}$ and $\mathrm{Y}$ scaling and categorized in 5 grades the result was grade $1 ; 13(25 \%)$ patients, grade 2; $20(40 \%)$ patients, grade $3 ; 13(25 \%)$ patients, grade $4 ; 5(10 \%)$ patients and we found no patient in grade 5 .

The mean pulse rate was $90 \pm 5.5$ beats per min irrespective of the gender and age group. The mean systolic blood pressures were $170 \pm 15$ and diastolic blood pressure was $90 \pm 5$.

Regarding the family history of $\mathrm{PD}$, the result was $10(20 \%)$ patients have no family history of PD and $41(80 \%)$ patients have no idea or do not remember or not certain of the existing of PD in the family (Table 1).

\subsection{Frequency and Symptoms of Autonomic Dysfunction}

In this study, 24 (47\%) patients (54\% male $46 \%$ female) had one or more symptoms of autonomic dysfunction (Table 2). The most common symptoms of autonomic dysfunction was constipation 16 (66\%) patients and bloating $16(66 \%)$ patients, exercise intolerance was seen next $13(54 \%)$ patients urinary incontinence 11 (46\%) patients, incomplete emptying of the bladder $10(42 \%)$ patients, loss of appetite $10(42 \%)$ patients, dizziness $7(29 \%)$ patients, fainting when the patient tries to stand in supine position $5(21 \%)$ patients, and lastly sweating abnormality $2(8 \%)$ patients (Figure 1 ). 
Table 1. Demographic and clinical characteristics of Parkinson disease patients.

\begin{tabular}{|c|c|c|}
\hline Variables & All cases, $\mathrm{n}=51$ & $P$ value \\
\hline Gender $(M / F), n$ & $31 / 20$ & 0.361 \\
\hline Age, years, Mean (SD) & $55 \pm 5$ & 0.001 \\
\hline Age at onset, years, Mean (SD) & $58(11.7)$ & 0.005 \\
\hline \multicolumn{3}{|l|}{ Age group, n (\%) } \\
\hline 40 - 50 years & $10(20 \%)$ & \\
\hline 51 - 60 years & $13(25 \%)$ & \\
\hline $61-70$ years & $16(31 \%)$ & \\
\hline Above 70 years & $12(24 \%)$ & \\
\hline Disease duration, years, Mean (SD) & $7.6(3.5)$ & 0.001 \\
\hline Less than 1 year, $\mathrm{n}(\%)$ & $11(21 \%)$ & \\
\hline $2-5$ years, $n(\%)$ & $23(45 \%)$ & \\
\hline $6-10$ years, $n(\%)$ & $11(22 \%)$ & \\
\hline More than 11 years, n (\%) & $6(12 \%)$ & \\
\hline \multicolumn{3}{|l|}{ A grade of Parkinson disease $n(\%)$} \\
\hline Grade 1 & $13(25 \%)$ & \\
\hline Grade 2 & $20(40 \%)$ & \\
\hline Grade 3 & $13(25 \%)$ & \\
\hline Grade 4 & $5(10 \%)$ & \\
\hline Grade 5 & $0(0 \%)$ & \\
\hline \multicolumn{3}{|l|}{ The family history of PD, $\mathrm{n}(\%)$} \\
\hline Yes & $0(0 \%)$ & \\
\hline No & $10(20 \%)$ & \\
\hline No recall & $41(80 \%)$ & \\
\hline $\mathrm{SBP}(\mathrm{mmHg})$, mean $(\mathrm{SD})$ & $170 \pm 15$ & \\
\hline DBP (mmHg), mean (SD) & $90 \pm 5$ & \\
\hline Pulse rate, mean (SD) & $90(5.5)$ & \\
\hline Current anti PD medication, Yes n (\%) & $40(78 \%)$ & 0.521 \\
\hline
\end{tabular}

$\mathrm{M} / \mathrm{F}=$ male/female; $\mathrm{SD}=$ stranded deviation, $\mathrm{SPB}=$ systolic blood pressure, $\mathrm{DBP}=$ diastolic blood pressure, $\mathrm{PD}=$ Parkinson disease. Values are $\mathrm{n}(\%)$ unless otherwise specified. The $\mathrm{P}$ values are based on unpaired t-test for age and $\chi^{2}$ test for other variables.

Table 2. Percentage of patients with Parkinson disease according to the presence of auto nomic dysfunction symptoms.

\begin{tabular}{ccc}
\hline Autonomic symptoms & Total number & Percentage \\
\hline Yes & 24 & $47 \%$ \\
No & 27 & $53 \%$ \\
Total number of patients & 51 & $100 \%$ \\
\hline
\end{tabular}




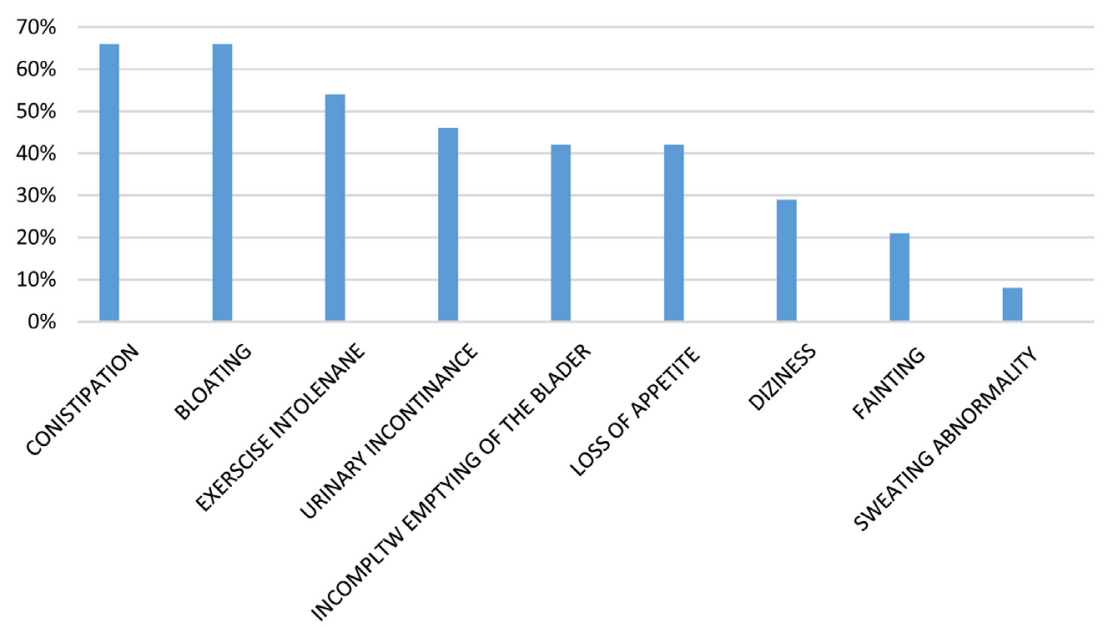

Figure 1. Frequency and percentage of autonomic dysfunction symptoms among Parkinson's disease patients.

The mean age of patients was of $59.3 \pm 10$, the age groups were $41-50$ years was $2(20 \%)$ patients, 51 - 60 years was $5(38 \%)$ and $61-70$ years was $9(56 \%)$ and above 70 years was $8(66 \%)(P=0.007)$ (Figure 2$)$.

The result of the Onset of PD duration was less than 1 year no patients, 2 to 5 years 9 of $23(39 \%)$ patients, 6 - 11 years 9 of 11 (82\%) patients and more than 11 years 6 of $6(100 \%)$ patients. the result showed a high significance $(P=0.007)$ (Figure 3).

The severity of the disease assessed using $\mathrm{H}$ and $\mathrm{Y}$ scaling compared the patients with autonomic dysfunction to the total number of patients with the same grade result was grade 1; no patients, grade 2; $8(40 \%)$ patients, grade 3; $11(84 \%)$ grade $4 ; 5$ (100\%) patients the pattern was shown (Figure 4).

Autonomic dysfunction symptoms and motors symptoms combined can cause trouble to the patient as in $46 \%$ [11] patients reported that. With only $4 \%$ [1] patient trouble most by the autonomic dysfunction alone while the motor symptom alone was much worse in 50\% [12] patients (Figure 5).

$46 \%$ (11 patients) had these symptoms constant with time while $50 \%$ [12] patients found it progressive and worsen over the time only $4 \%$ (1) patient reported that his symptoms were much better than before (Figure 6).

\subsection{Drugs and Treatments}

Prescribed drugs for the patients with PD were levodopa preparation, selegiline. The result showed that 20 (83\%) taking their anti PD medication despite developed the symptoms of dysautonomia and the relationships between the development of autonomic dysfunction and the respective drugs prescribed for PD was not statistically significant $(\mathrm{P}=0.46)$ (Table 3$)$.

\subsection{Risk Factors and Comorbidities}

Smoking was assessed in this study as a potential risk factor for developing the 


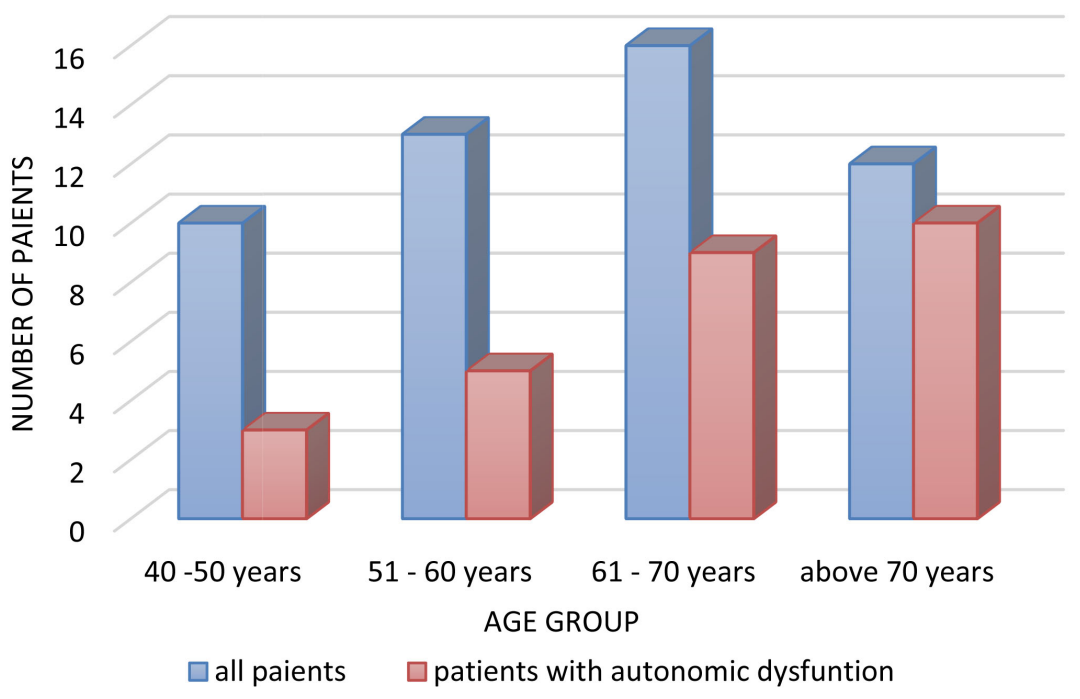

Figure 2. Comparison between the number of Parkinson disease patients with autonomic dysfunction and the total number of the patients distributed by age groups.

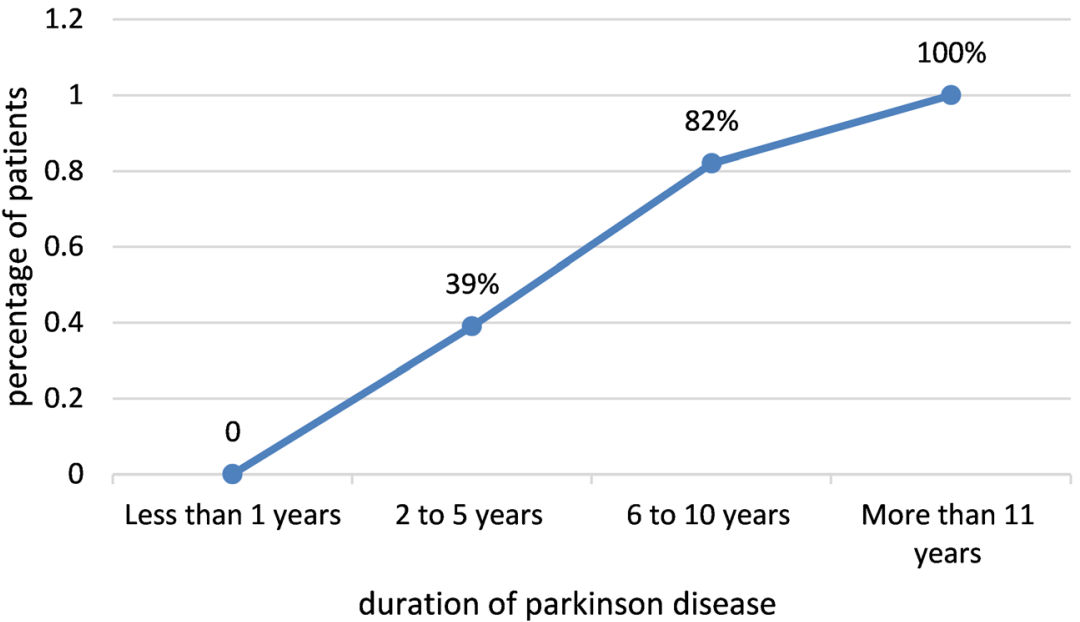

Figure 3. Displays relation between the autonomic dysfunction and duration of the disease in Parkinson disease patients.

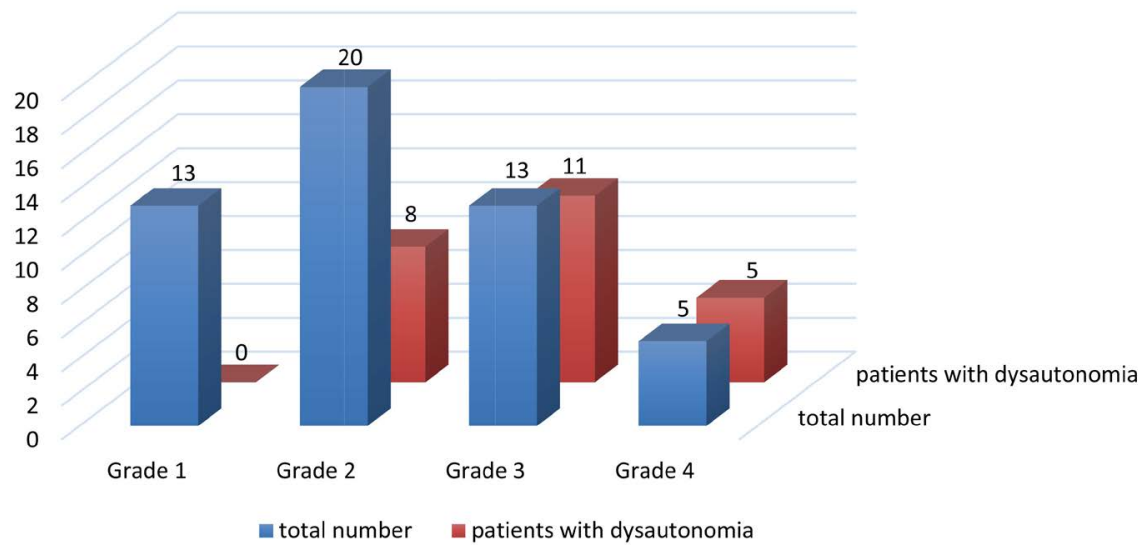

Figure 4. Total number of the patients compared to the patients with autonomic dysfunction distributed by grade of the disease. 


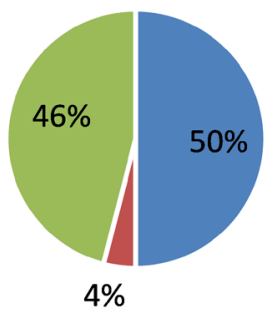

- MOTOR SYMPTOMS

- AUTONOMIC SYMPTOMS

- BOTH MOTOR AND AUTONOMIC

Figure 5. Percentage of the patients by the most annoying symptoms to the Parkinson's patient with autonomic dysfunction.

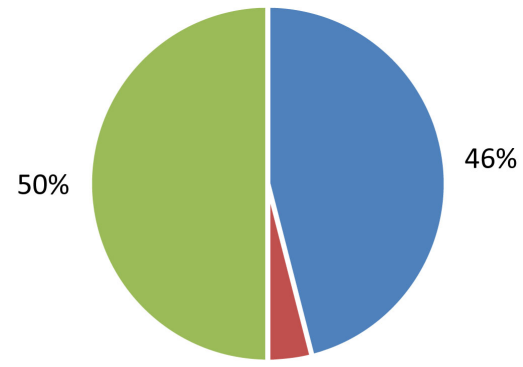

$4 \%$

- CONISTANT

- RELIEF WITH TIME

- PROGRESSED WITH TIME

Figure 6. The progression of autonomic dysfunction symptoms in Parkinson's disease patients.

Table 3. Percentage of Parkinson disease patients according to the current use of anti-Parkinson medication.

\begin{tabular}{cccc}
\hline Current anti PD medication & Total number & Patients with auto D & Percentage \\
\hline Patients taking their medication & 40 & 20 & $50 \%$ \\
Patients not taking their medication & 11 & 4 & $36 \%$ \\
Total number of patients & 51 & 24 & \\
\hline
\end{tabular}

$\mathrm{P}=0.422$.

autonomic dysfunction. 19 (37\%) patients were a smoker or had a history of smoking and 32 (62\%) patients had never smoked in their life. All the smoker were males. Statistical Analysis showed no significant differences between the patients who smoke and had the autonomic dysfunction and other patients who smoke and don't have autonomic dysfunction $(\mathrm{P}=0.3)$.

Also, body mass index was calculated with mean $24.5 \pm(1.5)$ in all patients and $23 \pm 2$ in a patient with autonomic dysfunction. the result has been categorized and grouped into three, overweight if BMI above 25 the result as flow; $20 \%$ (patient male 60\% female 40\%) were underweight and 80\% (41 patient 60 male 40 female) were within the range of healthy weight $(P=0.105)$. 
Chronic disease was explored and resulted in $30(59 \%)$ patients $(60 \%$ male $40 \%$ female) had hypertension and 5 (10\%) patients ( $80 \%$ male $20 \%$ female) had diabetes and $1(2 \%)$ patient has cardiac problem (IHD) and $30 \%$ has no chronic disease.

To estimate the potential risk factors of autonomic dysfunction in Parkinson's patients, multivariate LR analysis was performed in the entire sample, including the demographic parameters, disease onset duration and susceptible risk factors for developing the dysfunction which includes the history of smoking and presence of comorbidities like HTN, DM, and IHD. The result indicated that potential risk factors include age, duration of the disease, and stage of Parkinson disease significantly associated with the development of autonomic dysfunction (Table 4).

\section{Discussion}

Patients with Parkinson's disease (PD) have a combination of motor impairment, cognitive dysfunction and autonomic failure during their illness. This study, thought that autonomic dysfunction would be more severe in advanced PD patients than in early stages PD patients. For this purpose, several associate factors thought to affect the presence of autonomic dysfunction have been assessed and their possible correlation with each other in a population of PD patients.

A (51) patients with Parkinson's disease were investigated to identify the risk factors for developing symptoms of autonomic dysfunction. And it is found that $47 \%$ of these patients have developed one or more autonomic dysfunction symptoms. It is observed more commonly in the elderly above 61 years old which makes age the stronger risk of developing these symptoms.

The result of study support another one done In Nigeria in 2004 [12] which showed that $51.5 \%$ of PD developed autonomic dysfunction and concluded that Autonomic dysfunction was found to be common in Africans with PD, in contrast to [13] in Germany which was $38 \%$ in large sample size possibly genetic or environmental factors may contribute to this.

Male to female ratio was 1.5 to 1 respectively which follows the distribution of gender that has been described by local study in Parkinson disease [14] and international studies [3] and [15].

Symptoms of autonomic dysfunction correlate with the disease advances, therefore, the longer duration of the disease the higher chance of the symptoms

Table 4. Univariate logistic regression analysis for Parkinson disease autonomic dysfunction.

\begin{tabular}{ccccc}
\hline Variable & $\beta$ & S.E. & OR & $95 \%$ CI \\
\hline Age & 0.428 & 0.007 & 1.043 & $1.029-1.103$ \\
Disease Duration & 0.859 & 0.027 & 1.068 & $1.034-1.046$ \\
Stage of Parkinson Disease & 0.273 & 0.006 & 1.014 & $1.007-1.261$ \\
\hline
\end{tabular}


to manifest. In this study the symptoms manifest in the later stage of Parkinson patients comparing this to another study result [15] where the symptoms of autonomic dysfunction could be proceeding and manifest earlier than motor symptoms of Parkinson disease.

The most common symptoms of autonomic dysfunction in this study was constipation and bloating same as [16]. Gastrointestinal problems are common in elderly patients even without Parkinson disease but in the presence of the disease could exaggerate the symptom which becomes more obvious, another problem could explain this symptom is reduction in moving around which is vital for alimentary system physiology and function also lack interest in food and decrease the appetite which is also observed in $40 \%$ of patients in this study may all contribute to this presentation, an important factors, the drugs of anti-Parkinson's disease also have some role in the manifestation of this symptom as side effect of particular drug group.

Cardiovascular system has been affected and observed also in this study as intolerance to exercise around 54\% of patients compared to result in another study [7] which suggests that orthostatic hypotension is the commonest symptoms of Parkinson's disease.

Symptomatic orthostatic hypotension is significantly related to the duration of disease, and advanced age and maybe the daily anti PD medications dose. Cardiovascular involvement in autonomic dysfunction could attribute to tissue low perfusion because of orthostatic hypotension, defined as a sustained fall in BP of $20 \mathrm{~mm} \mathrm{Hg}$ systolic or $10 \mathrm{~mm} \mathrm{Hg}$ diastolic when moving from supine to standing. This phenomenon presents as dizziness and fainting which are observed in the result of this study.

Another crucial problem with autonomic failure in Parkinson's disease is that the occurrence of orthostatic hypotension during the disease makes the diagnosis of idiopathic Parkinson's disease uncertain and evokes other degenerative diseases such as multiple system atrophy.

Urinary symptoms as incontinence have high rate among patients in this study as $45 \%$ of the patients with the almost equal ratio between male and female this could be related to neurogenic detrusor over activity which is very common in Parkinson disease. Another symptom with High rate is the inability to complete emptying of the bladder $40 \%$ of patients. Together these symptoms cause a lot of discomfort to the patients.

Medication to treat PD might also have a clinical effect on autonomic function manifestation especial orthostatic hypotension with levodopa preparation and urinary symptoms with anticholinergic medication.

Smoking has been assessed in this study as an associate factor for developing the autonomic dysfunction which is not significantly observed a different between the patients who have the autonomic dysfunction and patients do not have it, in fact, smoking could delay the progression of the symptoms which has been seen in studies [4] and [17]. 
PD is known to cause low body weight this is multifactorial possibly due to anorexia and loss of appetite in general a gastrointestinal symptoms of the autonomic system has huge effect and expect to be observed in most of the patients in this study but only $12 \%$ of patients with autonomic dysfunction were underweight and showed no significance between the groups $(\mathrm{P}=0.105)$.

\section{Study limitation}

The results of the present study must be considering its limitations. First, the diagnosis of autonomic dysfunction in idiopathic Parkinson disease is based on clinical background and patients own history with no specific autonomic system test such as Valsalva maneuver and Head-up tilt test investigation to support the diagnosis. Other things due to an overlap of symptoms of PD with autonomic dysfunction and other Parkinsonism disorder one may argue that it may lead to inaccuracy and mislabel. However, in attempted to control this distorting by eliminating all unclear cases and cases with overlap, secondly it is to consider the small sample size has been used in this study as idiopathic Parkinson disease is rare. Last but not the least, the study is a cross-sectional and has a limitation in reflecting whether these risk factors contribute to developing the autonomic dysfunction or the latter influence these variables. But as mentioned above, this cross-section study may help to screen factors in the longitudinal study.

\section{Conclusion}

The study demonstrates that autonomic dysfunction is not only common in Sudanese PD, but it increases in severity with advancing in the disease stages; hence the advanced stage is, the greater chance to manifest the symptoms. Elder age with long disease duration is considered as some strong factor determining the presence of autonomic dysfunction.

\section{Acknowledgements}

I am deeply grateful to Professor Atif Elagib, national Centre for researchers who provided his practical advice.

I am indebted to Dr. Rasha Azrag, University of Khartoum science's college for her constructive advice during the preparation of the final manuscript. And Dr khattap Ibrahim for helping with the result output.

I also wish to express my sincere gratitude to the patients and their families who made this work possible.

\section{Conflicts of Interest}

The authors declare no conflicts of interest regarding the publication of this paper.

\section{References}

[1] Parkinson, J. (2002) An Essay on the Shaking Palsy. The Journal of Neuropsychiatry and Clinical Neurosciences, 14, 223-236. https://doi.org/10.1176/jnp.14.2.223 
[2] Braak, H., Ghebremedhin, E., Rüb, U., Bratzke, H. and Del Tredici, K. (2004) Stages in the Development of Parkinson's Disease-Related Pathology. Cell and Tissue Research, 318, 121-134. https://doi.org/10.1007/s00441-004-0956-9

[3] Miller, I.N. and Cronin-Golomb, A. (2010) Gender Differences in Parkinson's Disease: Clinical Characteristics and Cognition. Movement Disorders, 25, 2695-2703. https://doi.org/10.1002/mds.23388

[4] Morens, D.M., Grandinetti, A., Reed, D., White, L.R. and Ross, G.W. (1995) Cigarette Smoking and Protection from Parkinson's Disease: False Association or Etiologic Clue? Neurology, 45, 1041-1051. https://doi.org/10.1212/WNL.45.6.1041

[5] van der Marck, M.A., et al. (2012) Body Mass Index in Parkinson's Disease: A Meta-Analysis. Parkinsonism and Related Disorders, 18, 263-267.

https://doi.org/10.1016/j.parkreldis.2011.10.016

[6] Chaudhuri, K.R. (2001) Autonomic Dysfunction in Movement Disorders. Current Opinion in Neurology, 14, 505-511. https://doi.org/10.1097/00019052-200108000-00012

[7] Allcock, L.M. (2004) Frequency of Orthostatic Hypotension in a Community Based Cohort of Patients with Parkinson's Disease. Journal of Neurology, Neurosurgery, and Psychiatry, 75, 1470-1471. https://doi.org/10.1136/jnnp.2003.029413

[8] Cersosimo, M.G. and Benarroch, E.E. (2008) Neural Control of the Gastrointestinal Tract: Implications for Parkinson Disease. Movement Disorders, 23, 1065-1075. https://doi.org/10.1002/mds.22051

[9] Petrovitch, H., et al. (2009) Bowel Movement Frequency in Late-Life and Substantia Nigra Neuron Density at Death. Movement Disorders, 24, 371-376. https://doi.org/10.1002/mds.22360

[10] Campos-Sousa, R.N., Quagliato, E., da Silva, B.B., de Carvalho, R.M., Ribeiro, S.C. and de Carvalho, D.F.M. (2003) Urinary Symptoms in Parkinson's Disease: Prevalence and Associated Factors. Arquivos de Neuro-Psiquiatria, 61, 359-363. https://doi.org/10.1590/S0004-282X2003000300007

[11] Hoehn, M.M. and Yahr, M.D. (1967) Parkinsonism: Onset, Progression and Mortality. Neurology, 17, 427-442. https://doi.org/10.1212/WNL.17.5.427

[12] Okubadejo, N.U. and Danesi, M.A. (2004) Frequency and Predictors of Autonomic Dysfunction in Parkinson's Disease: A Study of African Patients in Lagos, Nigeria. Nigerian Postgraduate Medical Journal, 11, 45-49.

[13] Wüllner, U., et al. (2007) Autonomic Dysfunction in 3414 Parkinson's Disease Patients Enrolled in the German Network on Parkinson's Disease (KNP e.V.): The Effect of Ageing. European Journal of Neurology, 14, 1405-1408.

[14] Khalid, K., Hussien, A., Khalafela, K., Yonis, A., Eltoum, A. and Hassan, A. (2009) Clinical Presentation of Parkinson's Disease among Sudanese Patients. Sudan Journal of Medical Sciences, 4, 285-289. https://doi.org/10.4314/sjms.v4i3.48324

[15] Kaufmann, H., Nahm, K., Purohit, D. and Wolfe, D. (2004) Autonomic Failure as the Initial Presentation of Parkinson Disease and Dementia with Lewy Bodies. Neurology, 63, 1093-1095. https://doi.org/10.1212/01.WNL.0000138500.73671.DC

[16] Edwards, L.L., Pfeiffer, R.F., Quigley, E.M.M., Hofman, R. and Balluff, M. (1991) Gastrointestinal Symptoms in Parkinson's Disease. Movement Disorders, 6, 151-156. https://doi.org/10.1002/mds.870060211

[17] Hellenbrand, W., et al. (1997) Smoking and Parkinson's Disease: A Case-Control Study in Germany. International Journal of Epidemiology, 26, 328-339. https://doi.org/10.1093/ije/26.2.328 